\title{
Can't stop the craving: The effect of impulsivity on cue-elicited craving for alcohol in heavy and light social drinkers
}

\author{
Harilaos Papachristou • Chantal Nederkoorn • \\ Remco Havermans • Martje van der Horst • \\ Anita Jansen
}

Received: 18 October 2010 /Accepted: 18 February 2011 /Published online: 8 March 2011

(C) The Author(s) 2011. This article is published with open access at Springerlink.com

\begin{abstract}
Rationale A robust finding in the alcohol literature is that heavy and alcohol-dependent drinkers show stronger reactions to alcohol-related cues than light drinkers. However, there are individual differences in the degree of cue-elicited craving. Personality factors appear to be involved in cue reactivity and impulsivity is a possible candidate.

Objectives The aim of the present study was to examine the role of different aspects of impulsivity in heavy drinking and alcohol cue reactivity in social drinkers.

Methods Participants were heavy $(n=13)$ and light $(n=29)$ social drinkers who were exposed to neutral and alcoholrelated stimuli during a single laboratory session. Trait impulsivity, response inhibition, and sensitivity to reward were assessed with the Barratt Impulsiveness Scale (BIS-11), the Stop Signal Task, and the Card-Arranging Reward Responsivity Objective Test, respectively.

Results Heavy drinkers scored higher on trait impulsivity (BIS-11) than light drinkers. In addition, heavy drinkers reported elevated levels of craving for alcohol, but both in light and heavy drinkers, craving increased equally after exposure to alcohol cues. Impulsivity appeared to moderate this relation: heavy drinkers with ineffective response inhibition showed more craving to alcohol cues, compared to heavy drinkers with adequate response inhibition. In
\end{abstract}

H. Papachristou $(\square) \cdot$ C. Nederkoorn $\cdot$ R. Havermans $\cdot$

M. van der Horst $\cdot$ A. Jansen

Faculty of Psychology and Neuroscience, Maastricht University,

P.O. Box 616, 6200 MD Maastricht, The Netherlands

e-mail: h.papachristou@maastrichtuniversity.nl

H. Papachristou

U-Center,

Epen, The Netherlands light drinkers, response inhibition did not influence craving to alcohol cues.

Conclusions Different aspects of impulsivity are involved in heavy drinking and perhaps motivate alcohol consumption in a variety of ways. Having a deficient response inhibition appears to be a risk factor for heavy drinkers because it is associated with increased craving to alcohol cues.

Keywords Cue reactivity $\cdot$ Craving $\cdot$ Alcohol cue exposure $\cdot$ Impulsivity $\cdot$ Response inhibition $\cdot$ Sensitivity to reward

\section{Introduction}

Cue reactivity has been discussed extensively in the field of drug and alcohol abuse. A robust finding is that alcohol-dependent people relative to healthy controls show enhanced subjective (craving) and physiological reactivity (e.g. salivation) when exposed to alcoholrelated stimuli (Drummond 2000). Although several theories have been proposed to explain cue reactivity, most evidence favours a positive incentive account (Carter and Tiffany 1999; Drummond 2000). Regarding alcohol misuse/abuse, it is assumed that stimuli repeatedly paired with the reinforcing effects of alcohol acquire incentive value through classical conditioning and hence, elicit appetitive responses and promote drinking (Stewart et al. 1984; Robinson and Berridge 1993; Drummond 2000).

However, the relationship between cue reactivity and alcohol misuse/abuse seems to be more complicated than it was originally expected. After all, most people in western societies are frequently exposed to alcohol and alcoholrelated cues and one would expect that anybody who ever 
drinks alcohol should come to display cue-elicited craving. Nevertheless, not all of us become heavy or dependent drinkers and not everyone who has ever drunk alcohol will feel intense craving in the presence of alcohol-related cues. Approximately a third of the participants in alcohol cuereactivity studies do not react when exposed to alcoholrelated cues (Litt et al. 2000). Evidently, other factors mediate or moderate the relationship between cue reactivity and alcohol problem drinking. Some of these factors are conceptualized in terms of personality traits that may make someone vulnerable to substance misuse/abuse. A possible candidate is impulsivity.

Typically, impulsivity is conceptualized as a personality trait that leads to behaviour characterized by an inability to inhibit inappropriate action, lack of foresight or planning, and insensitivity to consequences (Dawe et al. 2004; Dawe and Loxton 2004; Reynolds et al. 2006; Dom et al. 2007). The concept of impulsivity has been incorporated into major personality theories including those by Eysenck (Eysenck and Eysenck 1977), Cloninger (1994), Zuckerman (1989), and Gray (1987), and many self-report instruments and behavioural tasks have been developed to measure it. However, the correlation between them is mostly weak (Dawe and Loxton 2004). Therefore, it seems that impulsivity is a multidimensional concept and different instruments and tasks assess different aspects of it.

There is wide agreement that there are at least two impulsivity dimensions. The first is related to response inhibition, while the second is concerned with motivation, in particular, sensitivity to reward (STR; Dawe et al. 2004; Guerrieri et al. 2008; Nederkoorn et al. 2009). The former refers either to the failure to suppress a prepotent response or to early responding due to incomplete evaluation of all the relevant information (Dawe et al. 2004; Dom et al. 2007; Guerrieri et al. 2008). The latter refers to a predisposition to detect and approach rewarding stimuli perhaps because of their increased salience (Gray 1987; Dawe et al. 2004; Guerrieri et al. 2008). Both impulsivity dimensions have been associated with alcohol problems.

As regards response inhibition, Noel et al. (2007) demonstrated that alcohol-dependent individuals versus healthy controls display impaired performance on the Go/No-Go task. Moreover, Nederkoorn et al. (2009) found that heavy drinking is associated with deficient performance on the Stop Signal Task, although only in women.

Regarding STR, Loxton and Dawe (2001) showed that increased STR is the best predictor of alcohol problems in adolescent girls. Similarly, Knyazev et al. (2004) found that self-reported STR predicts alcohol and substance use in adolescents and young adults. Finally, Kambouropoulos and Staiger (2007) reported that hazardous alcohol drinkers versus healthy controls score higher on self-report measures of STR.
Theoretically speaking, a high STR induces approach behaviour and increased cue reactivity to appetitive stimuli. In turn, these prepotent approach responses might not be inhibited in individuals with less effective response inhibition (Dawe et al. 2004). In particular, people high in STR may display intense craving to appetitive stimuli due to an overactive approach system, whereas people deficient in response inhibition may show increased craving to appetitive stimuli because they have difficulty inhibiting cueelicited approach responses.

Very few studies in the field of drug and alcohol addiction have examined the relationship between impulsivity and cue reactivity. Most of them have used self-report measures of impulsivity. Franken (2002) and Kambouropoulos and Staiger (2001) used the behavioural approach system (BAS) scale and found that increased STR is associated with high levels of craving for alcohol in dependent and heavy drinkers. Additionally, Doran et al. (2007, 2008) found that more impulsive smokers (impulsivity measured with the Barratt Impulsiveness Scale, BIS-11) show higher levels of cue reactivity than less impulsive smokers.

The aim of the present study is to examine the relationship between different dimensions of impulsivity and cue-elicited craving for alcohol in heavy and light social drinkers. First of all, it is hypothesized that heavy drinkers are more impulsive than light drinkers. Specifically, it is expected that heavy drinkers will score higher on measures of trait impulsivity and STR and will display poorer response inhibition relative to light drinkers. Furthermore, it is expected that a less efficient response inhibition and higher levels of STR are associated with higher levels of cue-elicited craving for alcohol in social drinkers.

\section{Methods}

\section{Participants}

Forty-two (32 women and 10 men) participants with a mean age of 26 years, $(\mathrm{SD}=9.66)$ volunteered to participate in the study. All participants were Dutch native speakers recruited from the community and the University of Maastricht through telephone interviews, emails, and advertisements. During recruitment, special care was taken to recruit heavy social drinkers.

Exclusion criteria were (a) any prescribed psychoactive medication, (b) any neurological, psychiatric and/or substance abuse disorders except for smoking tobacco, and (c) being pregnant. None of the participants was excluded on the basis of the above criteria.

All participants had been asked before the experiment via an email or a brief telephone interview about their preferred 
type/brand of alcoholic beverage. University students were rewarded for their participation in the experiment with either course credits or a gift certificate of 7.5€. Participants from the community were rewarded with a gift certificate of $10 €$ (which included travel expenses).

\section{Measures}

Barratt Impulsiveness Scale version 11 The Dutch version of the BIS-11 (Patton et al. 1995) is a 30-item self-report questionnaire that assesses trait impulsivity. Each item is reported on a four-point scale. The total score varies from 30 (low impulsivity) to 120 (high impulsivity). The BIS-11 consists of three factors: Attentional Impulsiveness, Motor Impulsiveness, and Non-planning Impulsiveness. The original BIS-11 has been demonstrated to be reliable in both clinical and non-clinical populations (Patton et al. 1995).

Response inhibition The stop signal task (SST) was used to assess response inhibition (Logan et al. 1997). Each trial in this task begins with a $500-\mathrm{ms}$ fixation point in the centre of the computer screen. Then, a go-trial follows. In each gotrial, participants are instructed to press as fast as possible either the right or the left "shift" button when a square pattern is presented either on the right or the left compartment of the screen, respectively. In $25 \%$ of the trials, an acoustic stop signal of $1,000 \mathrm{~Hz}$ is heard after the go-signal indicating that the participants must withhold their response.

The stop signal delay is initially set at $250 \mathrm{~ms}$ but throughout the task is adjusted dynamically allowing the participants to successfully inhibit their responses at approximately $50 \%$ of the stop trials. When the participant fails to withhold the response, the next stop signal is presented $50 \mathrm{~ms}$ earlier thereby, making the task easier. On the other hand, when the participant inhibits the response successfully, the next stop signal is presented $50 \mathrm{~ms}$ later, thereby making the task more difficult.

The dependent variable of interest in the task is the stop signal reaction time (SSRT), which is the difference between the mean Go reaction time and the mean stop signal delay, measured in milliseconds (ms). A higher SSRT is an index of impaired response inhibition.

In the present task, three practice blocks of, respectively, 6,12 , and 24 trials were followed by four test blocks of 64 trials each. Between blocks, participants were allowed to have a short break.

Sensitivity to reward STR was measured with the CardArranging Reward Responsivity Objective Test (CARROT; Al-Adawi and Powell 1997; Powell et al. 1996). The task involves four trials (T1-T4). During T1, participants have to sort 60 cards as fast as possible. Participants' performance time on $\mathrm{T} 1$ is a time limit for each of the following three trials (T2-T4) which involve the sorting of 100 cards each. Trials T2 and T4 contain no reward. However, in T3 participants are instantly rewarded with $0.20 €$ for every fifth card sorted correctly. The dependent variable is the difference between the number of cards sorted in the rewarded trial and the average number of cards sorted in the two non-rewarded trials [T3-(T2+T4)/2]. Scores are converted to rates by dividing the number of cards sorted in each trial by the time taken to sort the 60 cards at $\mathrm{T} 1$.

Craving Craving was measured with five items chosen from the shortened version of the Desires for Alcohol Questionnaire (Love et al. 1998). The five-item craving questionnaire consisted of those items with the highest loading on each of the following four factors: (1) strong desires and intentions to use alcohol (two items were chosen) (2) positive and negative reinforcement, (3) control over drinking, and (4) mild desires to drink (Love et al. 1998). The five-item questionnaire had a good internal consistency, with a Cronbach alpha coefficient of 0.79 .

AUDIT Alcohol problems were assessed with the Alcohol Use Disorders Identification Test (AUDIT; Fleming et al. 1991; Saunders et al. 1993). This selfreport instrument consists of ten multiple-choice items and is used to discriminate between people whose alcohol consumption is not harmful and those whose alcohol consumption reaches hazardous and problematic levels. A cut-off score of 11 was used in the present study (Fleming et al. 1991; Saunders et al. 1993).

Time-line follow-back questionnaire Alcohol use was assessed with a self-report instrument based on the timeline follow back-questionnaire (Sobell and Sobell 1990). Participants had to report how many alcohol drinks of different types they had consumed on each day for the last 30 days. The sum score in this questionnaire is the total amount of alcohol standard drinks consumed during the last 30 days.

\section{Procedure}

Ethical approval was sought and obtained from the Ethical Committee of the Psychology Faculty of Maastricht University. Participants took part in one individual testing session arranged between 11:00 am and 18:00 pm.

Participants first had to read and sign the informed consent form and then to complete a brief demographic questionnaire. Following this, STR and response inhibition were assessed with the CARROT and the SST, respectively, 
in a counterbalanced order. Having done this, participants were exposed first to water and later to their preferred type/ brand of alcoholic beverage for $3 \mathrm{~min}$, respectively. The order of cue-exposure conditions was not counterbalanced to avoid carry-over effects (Rohsenow and Niaura 1999). There was a 5-min break between the cue-exposure conditions during which the participant was left alone in the laboratory to relax and to read some magazines (no alcohol advertisements were present; Fig. 1).

At the beginning (before cues were present) and at the end of each cue-exposure condition (while the cues were still present), participants rated their craving levels (Fig. 1). During the alcohol exposure session, participants were presented with a commercially labelled bottle of their preferred brand of alcoholic beverage and a proper (for the type of beverage) empty glass. Then, they were asked to open the bottle and pour the alcoholic beverage into the glass. Following this, they were instructed to pick up the glass and sniff the alcoholic beverage, stare at the glass and the bottle of alcohol, sniff the alcoholic beverage in the bottle, and finally immerse their fingers into the glass with the alcoholic beverage and touch their lips and tongue with their fingers. In this way, they could taste the alcoholic beverage without drinking it. The instructions were repeated throughout the 3-min period of the exposure. The water exposure was identical to the alcohol exposure but the cue was a commercially labelled bottle of spring water. Drinking alcohol or water was not allowed during the exposure.

At the end of the alcohol exposure period, the Timeline Alcohol Questionnaire, the AUDIT, and the BIS-11 were administered to the participants in a counterbalanced order (Fig. 1). Finally, they were thanked, debriefed, and received the reward for their participation in the experiment.

\section{Statistical Analysis}

Based on their AUDIT scores with a cut-off score of 11, participants were divided into heavy $(N=13)$ and light $(N=$ 29) social drinkers. One-way independent analysis of variance (ANOVA) was used to compare the heavy and light drinking groups on craving and impulsivity measures. Moreover, a median split was conducted on the CARROT, SST, and BIS-11 scores and participants were classified as high and low on each of these impulsivity measures, leading to three 2 (drinking group: heavy vs. light drinkers) $\times 2$ (impulsivity: high vs. low in impulsivity) $\times 4$ (cue exposure: baseline water, water exposure, baseline alcohol, alcohol exposure) different repeated-measures ANOVAs. The drinking group and the impulsivity level were the between-subjects factors, while cue exposure (four time points: baseline water, water exposure, baseline alcohol and alcohol exposure) was the within-subject factor. Craving for alcohol was the dependent variable. Greenhouse-Geisser correction was applied when Mauchly's test of sphericity was significant.

\section{Results}

General characteristics of the participants

One-way independent ANOVA indicated that the two drinking groups differed significantly on the amount of alcohol consumed in the 30 days before participation, $F(1,40)=30.43, p<0.001$, with heavy drinkers $(M=102.69$, $\mathrm{SD}=53.94)$ drinking more than light drinkers $(M=31.97$, $\mathrm{SD}=29.34)$. Furthermore, there was a significant age difference between the two drinking groups, $F(1,40)=$ 4.59, $p<0.05$, with heavy drinkers $(M=20.92, \mathrm{SD}=2.06)$ being younger than light drinkers $(M=27.55, \mathrm{SD}=10.10)$. Finally, the gender distribution (male/female ratio) for each drinking group was $6 / 23$ and $4 / 9$ for light and heavy drinkers, respectively.

\section{Trait and behavioural impulsivity measures}

There was no significant difference in the $\operatorname{SST}(F[1,40]<1$, n.s. $)$ and CARROT $(F[1,40]<1$, n.s. $)$ performances between the two drinking groups. Furthermore, there was no correlation between the three impulsivity measures in the present study. However, heavy drinkers scored significantly higher in trait impulsivity (BIS-11) than light drinkers $(F[1,40]=6.13, p<0.02)$. Further analysis indicated that there was a significant difference in motor impulsiveness (BIS-11 Factor 2; $F[1,40]=5.21, p<0.05$ ) and non-planning impulsiveness (BIS-11 Factor 3; $F[1,40]=6.18, p<0.02)$ but not in attentional impulsiveness (BIS-11 Factor $1 ; F[1,40]=3.22$, n.s.) between the two drinking groups. Table 1 shows the SST, CARROT and BIS-11 mean scores and standard deviations for heavy and light drinkers, respectively.
Fig. 1 Schematic representation of the experimental procedure

\begin{tabular}{|c|c|c|c|c|}
\hline Impulsivity tasks & water exposure & rest & alcohol exposure & questionnaires \\
\hline Craving Craving Craving Craving
\end{tabular}


Table 1 Mean and standard deviation of impulsivity measures for heavy and light drinkers

\begin{tabular}{lcc}
\hline Impulsivity measures & $\begin{array}{l}\text { Heavy drinkers } \\
(n=13)\end{array}$ & $\begin{array}{l}\text { Light drinkers } \\
(n=29)\end{array}$ \\
\hline BIS-11 (Total) & $66.46(10.55) \mathrm{a}$ & $57.66(10.7) \mathrm{a}$ \\
BIS-11 (Attention) & $18.92(4.07) \mathrm{a}$ & $16.41(4.24) \mathrm{b}$ \\
BIS-11 (Motor) & $22.23(3.63) \mathrm{a}$ & $19.45(3.66) \mathrm{a}$ \\
BIS-11 (Non-planning) & $22.62(4.61) \mathrm{a}$ & $19.17(3.94) \mathrm{a}$ \\
SST & $220.90(54.68) \mathrm{a}$ & $222.67(50.02) \mathrm{b}$ \\
CARROT & $0.00(0.07) \mathrm{a}$ & $-0.02(0.07) \mathrm{b}$ \\
\hline
\end{tabular}

Means sharing similar letters within a row differ at $p<0.05$

BIS-11 Barratt Impulsiveness Scale version 11, SST Stop Signal Task, CARROT Card-Arranging Reward Responsivity Objective Test

The effects of response inhibition (SST) on craving during cue-exposure in heavy and light drinkers

A three-way repeated-measures ANOVA showed that there was a significant main effect of drinking group on craving levels $(F[1,38]=7.1, p<0.05$, Fig. 2). Inspection of Fig. 2 shows that heavy vs. light drinkers had higher craving levels at each time point of the cue-exposure.

The cue exposure had a significant main effect on craving, $(F[1.76,67]=22.96, p<0.001)$. Within-subjects simple contrasts showed that craving levels after the alcohol cue exposure were significantly higher than craving levels at each of the three previous time points of the cue exposure (alcohol exposure vs. water baseline, $F[1,38]=22.81, p<0.001$; alcohol exposure vs. water exposure, $F[1,38]=26.23, p<0.001$; alcohol exposure vs. alcohol baseline, $F[1,38]=35.4, p<0.001$; Fig. 2).

However, there was no difference between the two drinking groups in their reactivity to the alcohol cues. The

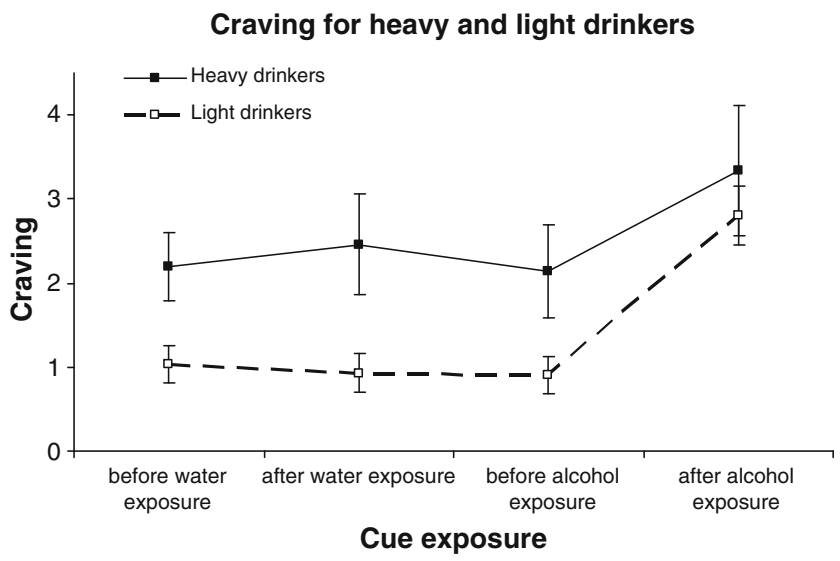

Fig. 2 Mean craving scores and S.E.M. of the two drinking groups (light vs. heavy drinkers) during the two cue-exposure conditions (water vs. alcohol) interaction between drinking group and cue exposure was not significant $(F[1.76,70]=1.1$, n.s. $)$. This means that the heavy drinkers did not report more cue-induced craving after the alcohol exposure than the light drinkers.

There appeared to be no significant main effect of response inhibition on craving $(F[1,38]=1.76$, n.s.). This means that, in general, participants high and low in response inhibition did not have different craving levels throughout the cue-exposure procedure.

More importantly, the analysis showed that response inhibition did influence reactivity to alcohol cues differently in heavy and light drinkers: there was a significant interaction between response inhibition, cue exposure and drinking group, on craving $(F[1.76,67]=4.2, p<0.05)$. Within-subjects simple contrasts (alcohol exposure was used as reference) indicated that the interaction was marginally significant for alcohol exposure vs. baseline alcohol $(F[1,38]=3.91, p=0.055)$ and significant for alcohol exposure vs. baseline water $(F[1,38]=5.75, p<0.05)$.

To further investigate this relationship, an ANOVA was performed between response inhibition and increase in craving (craving after alcohol exposure-craving before alcohol exposure) for each drinking group separately. The analysis showed that the interaction was marginally significant for the heavy drinkers $(F[1,11]=2.26$, $p=0.065$ ) but not for the light drinkers (Fig. 3). In other words, during the exposure to the alcohol cues, light drinkers with different response inhibition levels did not experience significantly different craving levels. On the other hand, heavy drinkers who were deficient in response inhibition experienced significantly higher craving than heavy drinkers with effective response inhibition. Therefore, the analysis demonstrated that ineffective response inhibition was associated with augmented cue-elicited craving in heavy drinkers but not in light drinkers. ${ }^{1}$

The effects of reward sensitivity and trait impulsivity on craving during cue-exposure in heavy and light drinkers

The same repeated-measures ANOVA was used, with groups based on the median score on the CARROT (high and low STR), heavy and light drinkers, and four time points during the cue exposure. The same significant main effects of drinking status, $(F[1,38]=5.98, p<0.05)$

\footnotetext{
${ }^{1}$ Based on a median split on monthly alcohol use, we can divide participants into equal groups of heavy $(N=21)$ and light $(N=21)$ drinkers. When using the median split the heavy drinking group is larger (21 vs. 13) and therefore the analysis has more power. When the same analysis is conducted, we find the same pattern of results with an even higher level of significance for the within-subjects simple contrasts (alcohol exposure vs. baseline alcohol, $F[1,38]=5.17, p<$ 0.05 ) and for the two-way interaction between response inhibition and alcohol cue exposure on the increase in craving for each drinking group separately, $F[1,19]=5.05, p<0.05$.
} 


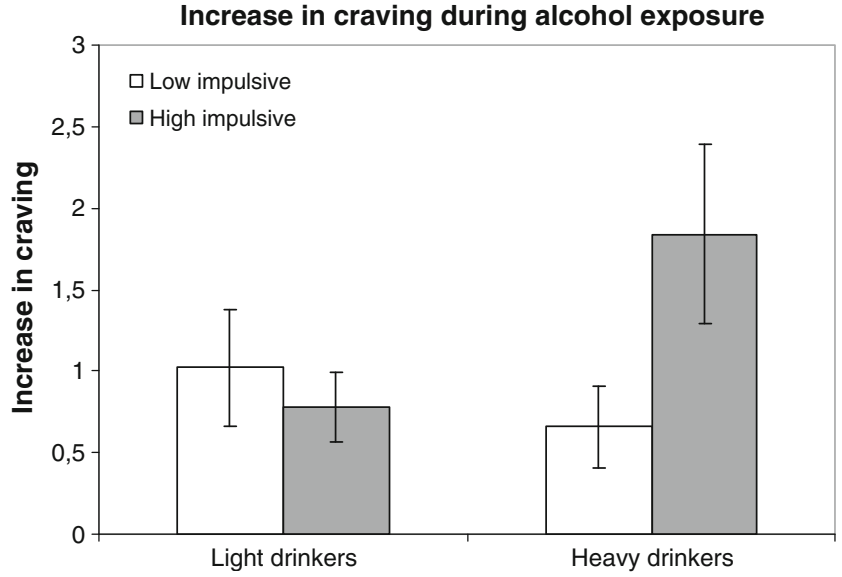

Fig. 3 Mean increase in craving and S.E.M. of the heavy and light drinkers according to their response inhibition levels (as measured with the SST) during the alcohol cue-exposure condition

and of cue exposure $(F[1.72,65.26]=19.26, p<0.001)$ were found as described above. However, neither the main effect of STR, nor any interaction effect appeared to be significant.

In addition, the 2 (heavy vs. light drinkers) $\times 2$ (low vs. high in trait impulsivity, based on median score on BIS-11) $\times 4$ (four time points during cue exposure) repeated-measures ANOVA showed again a marginally significant main effect of drinking status $(F[1,38]=4.02, p=0.052)$ and a highly significant main effect of cue exposure $(F[1.72,65.18]=$ $16.33, p<0.001)$ on craving. Neither the main effects of trait impulsivity nor any interaction effects were significant. ${ }^{2}$

\section{Discussion}

The primary aim of the present study was to investigate (a) whether there are differences in impulsivity between heavy and light social drinkers, (b) whether impulsivity moderates cue-elicited craving for alcohol in social drinkers. The results confirmed both of the above hypotheses.

First of all, heavy drinkers had higher impulsivity scores on the BIS-11 than light drinkers. Previous studies using the BIS-11 have also shown that heavy and dependent drinkers are more impulsive than healthy controls (Carlson et al. 2010; Lawrence et al. 2009; Rubio et al. 2008). As the BIS-11 is thought to be a measure of trait impulsivity, the present results imply that impulsivity, as a personality characteristic, may predispose an individual to develop heavy drinking. Specifically, the Non-planning Impulsiveness subscale assesses lack of reflection and self-control as well as a tendency to focus on the present, while the Motor Impulsiveness subscale reflects the tendency to act on the

\footnotetext{
$\overline{{ }^{2} \text { When we add }}$ age as a covariate, the pattern of all results remained the same and age was never significant as a covariate.
}

spur of the moment without consideration of the negative consequences of one's own behaviour (Carlson et al. 2010; Stanford et al. 2009). These attitudes may predispose an individual to heavy drinking perhaps in order to experience the immediate positive reinforcing qualities of alcohol without consideration of the future negative consequences of heavy drinking (e.g. dangers of binge drinking). Individuals high on these traits may have greater difficulty refraining from alcohol, once they find themselves in a situation where alcohol is available.

An alternative explanation could be that heavy drinking causes or exacerbates impulsive characteristics in an individual (Jentsch and Taylor 1999). In support of this argument, Scaife and Duka (2009) have shown that binge drinking is related to prefrontal cortex dysfunction in young social binge drinkers. Furthermore, Spinella (2005) found a negative correlation between the BIS-11 Non-planning Impulsiveness scale and a self-report index of the dorsolateral prefrontal cortex (DLPFC) and striatum activity (Spinella 2005; Stanford et al. 2009). Similarly, a negative correlation was reported between the BIS-11 Motor Impulsiveness subscale and a self-report index of orbitofrontal cortex activity (Spinella 2005; Stanford et al. 2009). It could be argued that heavy drinking patterns affect prefrontal cortex function and this was reflected on the higher BIS-11 scores of the heavy drinkers as compared to the scores of the light drinkers.

As regards the second hypothesis of the present study, the results showed that response inhibition, as measured with the SST, is associated with cue reactivity (craving) in heavy but not light social drinkers. It was found that heavy drinkers who were deficient in response inhibition had higher craving levels for alcohol than heavy drinkers who were efficient in response inhibition. Thus, a poorer response inhibition was associated with a difficulty to overcome strong appetitive-conditioned responses (e.g. cue-elicited craving for alcohol) in the presence of alcohol-related cues (e.g. a bottle of one's favourite alcoholic beverage). However, in our sample of young social drinkers, we found no evidence for the assumptions that heavy drinking results necessarily in impaired response inhibition or that response inhibition leads inevitably to heavy drinking. Therefore, it appears that a deficient response inhibition is a predisposing tendency or a risk factor that, in combination with a heavy drinking pattern and in the right context (e.g. in the presence of alcohol-related cues), leads to an inability to inhibit a conditioned appetitive response like craving.

Response inhibition is a prefrontal executive function and performance on the SST relies heavily on the right inferior prefrontal cortex and the frontostriatal pathway (Aron et al. 2004; Aron and Poldrack 2006; Rieger et al. 2003). From a psychological point of view, response 
inhibition, as measured with the SST, is concerned with the inhibition and cancellation of a well-learned motor response. However, evidence is accumulating that the same inhibitory mechanism may be involved in the inhibition of memories and affective responses (Aron et al. 2004). Cue-elicited craving for alcohol is such an affective response that also relies on prefrontal activity. Neuroimaging studies show activation of a variety of prefrontal cortical areas and frontal subcortical structures in alcohol-dependent individuals during exposure to alcohol-related stimuli ( $\mathrm{Li}$ et al. 2009). As such, cueelicited craving and response inhibition may rely on common prefrontal neural pathways and interact with each other. Our results suggest that such an interrelationship is plausible. Further support for this assumption comes from the findings of a recent neuroimaging fMRI study with alcohol-dependent individuals who performed the SST (Li et al. 2009). Although also in this study there was no difference between the SSRTs of patients and healthy controls, effective inhibitors in the healthy control group showed stronger activation of the left DLPFC than effective inhibitors in the patient group ( $\mathrm{Li}$ et al. 2009). More importantly, within the patient group, those with higher craving levels showed more impaired DLPFC activation than those patients with lower craving levels (Li et al. 2009). Despite these preliminary results, more research is needed in order to understand this relationship at a psychological and neuropsychological level.

The current finding that heavy drinkers did not differ as a group in their SST performance from the light drinkers may result from the characteristics of the present sample and/or a moderate sample size. It is also possible that chronic alcohol abuse and dependence exacerbate inhibitory impairments in alcohol-dependent individuals and make it easier for these impairments to be detected by the SST in clinical populations.

Regarding reward sensitivity, the heavy drinkers did not differ in their CARROT performance from the light drinkers and no interaction was found between craving and reward sensitivity. At first glance, these findings appear to be in contrast with the results of previous studies (Franken 2002; Kambouropoulos and Staiger 2001). However, in earlier alcohol studies, STR was measured with a self-report scale (BAS), while in the present study we used the CARROT, which is a behavioural measure of STR (Franken 2002; Kambouropoulos and Staiger 2001). Although there is evidence for a relationship between trait reward sensitivity and CARROT performance, it may be that behavioural and self-report measures assess somewhat different aspects of STR (Dawe et al. 2004; Kambouropoulos and Staiger 2007). Therefore, in the present study, we cannot draw any definite conclusion regarding reward sensitivity because our measures may not have been sensitive enough or appropriate to detect any differences between the drinking groups or any relationship between craving and reward sensitivity.

In sum, our results show that different aspects of impulsivity are involved in heavy drinking and perhaps motivate alcohol consumption in a variety of ways. High trait impulsivity, as measured with the BIS-11, appears to be a personality characteristic of heavy social drinkers and may result in/from heavy drinking patterns in young social drinkers. More importantly, response inhibition, as measured with the SST, appears to be a risk factor for some heavy drinkers because it affects their craving and perhaps their alcohol consumption in the presence of alcohol-related cues. This, in turn, may lead to the development of more serious alcohol problems for some heavy drinkers.

It remains of course to investigate empirically if response inhibition affects not only the craving but also the drinking behaviour of social drinkers in the presence of alcohol-related cues. Moreover, it would be interesting to examine whether the same mechanisms apply to clinical populations. Finally, if response inhibition is a risk factor for heavy drinking, then training response inhibition would lead to less craving and perhaps less drinking for some heavy alcohol and problem drinkers. Indeed, evidence supporting this idea comes from a recent study by Houben et al. (submitted) who reported a decrease in heavy drinkers' alcohol consumption after a week of response inhibition training with a Go/No-Go task. Therefore, the early detection of response inhibition problems and the incorporation of response inhibition training techniques in therapeutic programs may be of great value for some problem drinkers.

Open Access This article is distributed under the terms of the Creative Commons Attribution Noncommercial License which permits any noncommercial use, distribution, and reproduction in any medium, provided the original author(s) and source are credited.

\section{References}

Al-Adawi S, Powell JH (1997) The influence of smoking on reward responsiveness and cognitive functions: a natural experiment. Addiction 92:1773-1782

Aron AR, Poldrack RA (2006) Cortical and subcortical contributions to stop-signal response inhibition: role of the subthalamic nucleus. J Neurosci 26:2424-2433

Aron AR, Robbins TW, Poldrack RA (2004) Inhibition and the right inferior frontal cortex. Trends Cogn Sci 8:170-177

Carlson SR, Johnson SC, Jacobs PC (2010) Disinhibited characteristics and binge drinking among university student drinkers. Addict Behav 35:242-251

Carter BL, Tiffany ST (1999) Meta-analysis of cue-reactivity in addiction research. Addiction 94:327-340 
Cloninger CR (1994) Temperament and personality. Curr Opin Neurobiol 4:266-273

Dawe S, Loxton NJ (2004) The role of impulsivity in the development of substance use and eating disorders. Neurosci Biobehav Rev 28:343-351

Dawe S, Gullo MJ, Loxton NL (2004) Reward drive and rash impulsiveness as dimensions of impulsivity: implications for substance misuse. Addict Behav 29:1389-1405

Dom G, De Wilde B, Hulstijn W, Sabbe B (2007) Dimensions of impulsive behaviour in abstinent alcoholics. Pers Individ Differ 42:465-476

Doran N, Spring B, McChargue D (2007) Effects of impulsivity on craving and behavioural reactivity to smoking cues. Psychopharmacology 194:279-288

Doran N, McChargue D, Spring B (2008) Effect of impulsivity on cardiovascular and subjective reactivity to smoking cues. Addict Behav 33:167-172

Drummond DC (2000) Human models in craving research: what does cue-reactivity have to offer clinical research? Addiction 95: S129-S144

Eysenck SBG, Eysenck HJ (1977) The place of impulsiveness in a dimensional system of personality description. Brit J Soc Clin Psychol 16:57-68

Fleming MF, Barry KL, MacDonald R (1991) The Alcohol Use Disorders Identification Test (AUDIT) in a college sample. Int $\mathbf{J}$ Addict 26:1173-1185

Franken IHA (2002) Behavioural approach system (BAS) predicts alcohol craving. Pers Individ Differ 32:349-355

Gray JA (1987) Perspectives on anxiety and impulsivity: a commentary. J Res Pers 21:493-509

Guerrieri R, Nederkoorn C, Jansen A (2008) The effect of an impulsive personality on overeating and obesity: current state of affairs. Psychol Topics 17:265-286

Jentsch DJ, Taylor JR (1999) Impulsivity resulting from frontostriatal dysfunction in drug abuse: implications for the control of behaviour by reward-related stimuli. Psychopharmacology 146:373-390

Kambouropoulos N, Staiger PK (2001) The influence of sensitivity to reward on reactivity to alcohol-related cues. Addiction 96:11751185

Kambouropoulos N, Staiger PK (2007) Personality, behavioural and affective characteristics of hazardous drinkers. Pers Individ Differ 42:213-224

Knyazev GG, Slobodskaya HR, Kharchenko II, Wilson GD (2004) Personality and substance use in Russian youths: the predictive and moderating role of behavioural activation and gender. Pers Individ Differ 37:827-843

Lawrence AJ, Luty J, Bogdan NA, Sahakian BJ, Clark L (2009) Impulsivity and response inhibition in alcohol dependence and problem gambling. Psychopharmacology 207:163-172

Li CR, Luo X, Yan P, Bergquist K, Sinha R (2009) Altered impulse control in alcohol dependence: neural measures of stop signal performance. Alcohol Clin Exp Res 33:740-750

Litt MD, Cooney NL, Morse P (2000) Reactivity to alcohol-related stimuli in the laboratory and in the field: predictors of craving in treated alcoholics. Addiction 95:889-900
Logan GD, Schachar RJ, Tannock R (1997) Impulsivity and inhibitory control. Psychol Sci 8:60-64

Love A, James D, Willner P (1998) A comparison of two alcohol craving questionnaires. Addiction 93:1091-1102

Loxton NL, Dawe S (2001) Alcohol abuse and dysfunctional eating in adolescent girls: the influence of individual differences in sensitivity to reward and punishment. Int J Eat Disord 29:455462

Nederkoorn C, Baltus M, Guerrieri R, Wiers RW (2009) Heavy drinking is associated with deficient response inhibition in women but not in men. Pharmacol Biochem Behav 93:331-336

Noel X, Van den Linden M, d'Acremont M, Bechara A, Dan B, Hanak C et al (2007) Alcohol cues increase cognitive impulsivity in individuals with alcoholism. Psychopharmacology 192:291298

Patton JH, Stanford MS, Barratt ES (1995) Factor structure of the Barratt Impulsiveness Scale. J Clin Psychol 51:768-774

Powell JH, Al-Adawi S, Morgan J, Greenwood RJ (1996) Motivational deficits after brain injury: effects of bromocriptine in 11 patients. J Neurol Neurosurg Psychiatry 60:416-421

Reynolds B, Ortengren A, Richards JB, de Wit H (2006) Dimensions of impulsive behaviour: personality and behavioural measures. Pers Individ Differ 40:305-315

Rieger M, Gauggel S, Burmeister K (2003) Inhibition of ongoing responses following frontal, nonfrontal, and basal ganglia lesions. Neuropsychology 17:272-282

Robinson TE, Berridge KC (1993) The neural basis of drug craving: an incentive-sensitization theory of addiction. Brain Res Brain Res Rev 18:247-291

Rohsenow DJ, Niaura RS (1999) Reflections on the state of cuereactivity theories and research. Addiction 94:341-351

Rubio G, Jimenez M, Rodrigo-Jimenez R, Martinez I, Avila C, Ferre F et al (2008) The role of behavioural impulsivity in the development of alcohol dependence: a 4-year follow-up study. Alcohol Clin Exp Res 32:1681-1687

Saunders JB, Aasland OG, Babor TF, De La Fuente JR, Grant M (1993) Development of the Alcohol Use Disorders Identification Test (AUDIT): WHO collaborative project on early detection of persons with harmful alcohol consumption-II. Addiction 88:791804

Scaife JC, Duka T (2009) Behavioural measures of frontal lobe function in a population of young social drinkers with binge drinking pattern. Pharmacol Biochem Behav 93:354-362

Sobell LC, Sobell MB (1990) Self-reports across addictive behaviours: issues and future directions in clinical and research settings. Behav Assess 12:77-90

Spinella M (2005) Self-rated executive function: development of the executive function index. Int J Neurosci 115:649-667

Stanford MS, Mathias CW, Dougherty DM, Lake SL, Anderson NE, Patton JH (2009) Fifty years of the Barratt Impulsiveness Scale: an update and review. Pers Individ Differ 47:385-395

Stewart J, de Wit H, Eikelboom R (1984) Role of unconditioned and conditioned drug effects in the self-administration of opiates and stimulants. Psychol Rev 91:251-268

Zuckerman M (1989) Personality in the third dimension: a psychobiological approach. Pers Individ Differ 10:391-418 